\title{
CaMKIV Signaling Is Not Essential for the Maintenance of Intrinsic or Synaptic Properties in Mouse Visual Cortex
}

\author{
(D) Nicholas F. Trojanowski and Gina G. Turrigiano \\ https://doi.org/10.1523/ENEURO.0135-21.2021 \\ Department of Biology, Brandeis University, Waltham, MA 02454
}

\begin{abstract}
Pyramidal neurons in rodent visual cortex homeostatically maintain their firing rates in vivo within a target range. In young cultured rat cortical neurons, $\mathrm{Ca}^{2+}$ /calmodulin-dependent kinase IV (CaMKIV) signaling jointly regulates excitatory synaptic strength and intrinsic excitability to allow neurons to maintain their target firing rate. However, the role of CaMKIV signaling in regulating synaptic strength and intrinsic excitability in vivo has not been tested. Here, we show that in pyramidal neurons in visual cortex of juvenile male and female mice, CaMKIV signaling is not essential for the maintenance of basal synaptic or intrinsic properties. Neither CaMKIV conditional knock-down nor viral expression of dominant negative CaMKIV (dnCaMKIV) in vivo disrupts the intrinsic excitability or synaptic input strength of pyramidal neurons in primary visual cortex (V1), and CaMKIV signaling is not required for the increase in intrinsic excitability seen following monocular deprivation (MD). Viral expression of constitutively active CaMKIV (caCaMKIV) in vivo causes a complex disruption of the neuronal input/output function but does not affect synaptic input strength. Taken together, these results demonstrate that although augmented in vivo CaMKIV signaling can alter neuronal excitability, either endogenous CaMKIV signaling is dispensable for maintenance of excitability, or impaired CaMKIV signaling is robustly compensated.
\end{abstract}

Key words: CaMKIV; firing rate homeostasis; homeostatic plasticity; intrinsic excitability; synaptic strength

\section{Significance Statement}

Homeostatic regulation of neuronal activity is essential for stable network function. However, little is known about the mechanisms that coordinate intrinsic and synaptic properties to regulate activity levels in vivo. $\mathrm{Ca}^{2+} /$ calmodulin-dependent kinase IV (CaMKIV) signaling regulates synaptic strength and intrinsic excitability in cultured neurons, but its role in these processes in vivo has never been tested. Here, we show that while CaMKIV signaling can modulate intrinsic excitability, it does not play an essential role in maintenance of intrinsic or synaptic properties in vivo.

Received March 31, 2021; accepted May 6, 2021; First published May 17, 2021.

The authors declare no competing financial interests.

Author contributions: N.F.T. and G.G.T. designed research; N.F.T. performed research; N.F.T. analyzed data; N.F.T. wrote the paper.

This work was supported by National Institutes of Health Grants NS R35111562 (to G.G.T.) and NS F32101832 (to N.F.T.).

Correspondence should be addressed to Gina G. Turrigiano at turrigiano@ brandeis.edu.

https://doi.org/10.1523/ENEURO.0135-21.2021

Copyright $\odot 2021$ Trojanowski and Turrigiano

This is an open-access article distributed under the terms of the Creative Commons Attribution 4.0 International license, which permits unrestricted use, distribution and reproduction in any medium provided that the original work is properly attributed.

\section{Introduction}

Homeostatic plasticity bidirectionally constrains neuronal activity to a target range by adjusting the relationship between neuronal inputs and firing rate (Turrigiano, 2012; Davis, 2013). Across a variety of vertebrate and invertebrate systems, homeostatic compensatory mechanisms overcome prolonged experimentally-induced changes in activity to return neuronal or network output to a baseline level (Chub and O'Donovan, 1998; Thoby-Brisson and Simmers, 1998; Burrone et al., 2002; Sakurai and Katz, 2009; Harley et al., 2015). The two most studied forms of homeostatic plasticity in the mammalian cortex are 
synaptic scaling and plasticity of intrinsic excitability (Turrigiano et al., 1998; Desai et al., 1999). During synaptic scaling, the strengths of all synapses onto a neuron are multiplicatively renormalized (Turrigiano and Nelson, 2004), while plasticity of intrinsic excitability modulates the excitability of the neuron as a whole primarily by modulating the abundance and biophysical properties of ion channels (Zhang and Linden, 2003). In rodent primary visual cortex (V1), monocular deprivation (MD) during the critical period first causes a decrease in firing rate in excitatory neurons responding to the deprived eye, followed by a homeostatic return to each neuron's own original firing rate (Hengen et al., 2016), demonstrating that each excitatory neuron has its own firing rate set point. Synaptic scaling and plasticity of intrinsic excitability can work cooperatively to homeostatically adjust the relationship between neuronal input and output, suggesting that they are jointly regulated (Lambo and Turrigiano, 2013). However, the signaling pathways that allow neurons to coordinate intrinsic and synaptic parameters to maintain these cell-specific firing rate set points in vivo are not well understood.

In cultured neurons, $\mathrm{Ca}^{2+} /$ calmodulin-dependent kinase (CaMK) signaling modulates baseline firing rate by jointly regulating synaptic scaling and intrinsic plasticity (Ibata et al., 2008; Goold and Nicoll, 2010; Joseph and Turrigiano, 2017). CaMK signaling links membrane $\mathrm{Ca}^{2+}$ flux to gene transcription, and the rate limiting step in this pathway, phosphorylation of the transcription factor CREB by CaMKIV, is constrained by the amount of CaMKIV in each neuron (Ma et al., 2014; Cohen et al., 2016). Decreasing CaMKIV signaling in vitro, mimicking decreased membrane $\mathrm{Ca}^{2+}$ entry due to decreased activity, causes synaptic upscaling and increased intrinsic excitability, while increasing CaMKIV signaling has opposing effects. The net result of these coordinated changes in miniature excitatory postsynaptic current (mEPSC) amplitude and intrinsic excitability is a shift in the firing rate of these neurons, arguing that CaMKIV signaling is involved in the regulation of firing rate set points in young neurons in vitro (Joseph and Turrigiano, 2017). CaMKIV signaling has also been implicated in the homeostatic regulation of the duration of action potentials that can follow prolonged hyperpolarization in vitro (Li et al., 2020). However, role of in vivo CaMKIV signaling in regulating synaptic strengths and intrinsic excitability in juvenile animals, at a developmental time when homeostatic plasticity is especially prominent, has not been explored.

Here, we tested if in vivo CaMKIV regulates mEPSC amplitude and intrinsic excitability. Using conditional CaMKIV knock-down, we found that decreasing CaMKIV protein levels during the critical period did not alter intrinsic excitability or mEPSC amplitude or frequency in excitatory neurons in layer (L)2/3 or L4 of monocular V1 (V1m), nor did it affect the homeostatic response of pyramidal neurons in V1m or binocular V1 (V1b) to MD. Dominant negative CaMKIV (dnCaMKIV) also failed to alter intrinsic excitability or synaptic properties, while constitutively active CaMKIV (caCaMKIV) caused a complex disruption of intrinsic excitability but did not affect
mEPSC frequency or amplitude. These results suggest that CaMKIV signaling is not essential for the regulation of baseline mEPSC properties or intrinsic excitability in the juvenile mouse visual cortex, but do not rule out a role for CaMKIV signaling in acutely modulating intrinsic excitability.

\section{Materials and Methods}

\section{Mice}

All experimental procedures used in this study were approved by the Institutional Animal Care and Use Committee at Brandeis University and followed the guidelines of the National Institutes of Health. Male and female B6.129-Camk $4^{\text {tm1Gsc }} /$ leg mice (EMMA EM:02126, RRID: IMSR_EM:02126) housed in groups were used for all experiments except where noted. In those cases, B6.129Camk $4^{\text {tm1Gsc}} / \mathrm{leg}$ mice were crossed with B6.129S2-

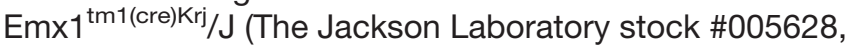
RRID:IMSR_JAX:005628) mice. All experiments began between zeitgeber time (ZT)0 and ZT4, and concluded by ZT10.

\section{Virus construction}

AAV9-CamKII-HI-eGFP-Cre-WPRE-SV40 (AV-9-PV2521) and AAV9-CamKII0.4-eGFP-WPRE-rBG (AV-9-PV1917) were purchased from the UPenn Vector Core. AAV9-CaMKII0.4-dnCaMKIV-GFP-3xNLS-WPRE and AAV9-CaMKII0.4-caCaMKIV-GFP-3xNLS-WPRE were constructed by replacing the CMV promotor in pEGFPCaMKIV-CA-Nuc and pEGFP-CaMKIV-DN-Nuc (Schmitt et al., 2004; Wayman et al., 2004) with a $0.4 \mathrm{~kb}$ CaMKII promoter fragment (Prakash et al., 2012), then ligating the constructs into an AAV9-MCS backbone (gift from Yasuyuki Shima and Sacha Nelson).

\section{Virus injection}

Stereotactic injection at postnatal day $(\mathrm{P}) 15$ or $\mathrm{P} 16$ was performed by anesthetizing mice using a ketamine/xylazine/acepromazine mixture, then injecting $200 \mathrm{nl}$ of AAV (diluted 1:10 from titer) from a glass micropipette pulled to a fine point. Injection coordinates $(0.5 \mathrm{~mm}$ rostral from lambda, $2.5 \mathrm{~mm}$ lateral from midline) were scaled to the measured lambda-bregma distance.

\section{Lid sutures}

Lid sutures were performed on P22 or P23. Animals were anesthetized with a ketamine/xylazine/acepromazine mixture, then the right eyelid was trimmed to promote fusion of the top and bottom eyelids during suturing. Eyelids were then sutured together with four 6-0 nylon sutures. Suture integrity was confirmed daily; only animals with intact sutures were used for experiments. For all MD experiments, animals that were anesthetized but not subject to lid suturing (sham) were used as controls.

\section{Acute slice preparation}

At the ages indicated for each experiment (ranging from P22 to P29), animals were deeply anesthetized with 
isoflurane, then rapidly decapitated. The brain was rapidly removed, then the region containing V1 was cut into 300$\mu \mathrm{m}$-thick coronal sections on a vibratome in carbogenated $\left(95 \% \mathrm{O}_{2}, 5 \% \mathrm{CO}_{2}\right)$ standard artificial CSF (ACSF; $126 \mathrm{~mm} \mathrm{NaCl}, 25 \mathrm{~mm} \mathrm{NaHCO}_{3}, 3 \mathrm{~mm} \mathrm{KCl}, 2 \mathrm{~mm} \mathrm{CaCl}_{2}$, 2 $\mathrm{mm} \mathrm{MgSO}_{4}, 1 \mathrm{~mm} \mathrm{NaH} \mathrm{PO}_{4}$, and $0.5 \mathrm{~mm}$ Na-ascorbate, osmolarity adjusted to $310 \mathrm{mOsm}$ with dextrose, $\mathrm{pH}$ 7.35). After slicing, slices were immediately transferred to a $34^{\circ} \mathrm{C}$ chamber filled with a continuously carbogenated choline-based solution (110 mm choline-Cl, $25 \mathrm{~mm}$ $\mathrm{NaHCO}_{3}, 11.6 \mathrm{~mm} \mathrm{Na}$-ascorbate, $7 \mathrm{~mm} \mathrm{MgCl}$, $3.1 \mathrm{~mm}$ Na-pyruvate, $2.5 \mathrm{~mm} \mathrm{KCl}, 1.25 \mathrm{~mm} \mathrm{NaH}_{2} \mathrm{PO}_{4}$, and 0.5 $\mathrm{mm} \mathrm{CaCl}$, adjusted to $310 \mathrm{mOsm}$ with dextrose, $\mathrm{pH}$ 7.35) for $5 \mathrm{~min}$, then transferred back to warm standard ACSF for $30 \mathrm{~min}$ (Ting et al., 2014). Slices were then moved to room temperature. Electrophysiological recordings were performed within $6 \mathrm{~h}$ of slicing.

\section{Electrophysiology}

In acute slices, V1m and V1b were identified by the shape and morphology of the white matter. Pyramidal neurons were targeted and identified by their teardrop shaped soma and apical dendrite, and their morphology and layer were confirmed post hoc by biocytin fills. Borosilicate recording electrodes with tip resistance between 4 and $8 \mathrm{M} \Omega$ were pulled on a Sutter P-97 micropipette puller. All recordings were performed on submerged slices continuously perfused with carbogenated $34^{\circ} \mathrm{C}$ standard ACSF with blockers as indicated below. Neurons were visualized on an Olympus BX51QWI upright epiflouresence microscope with a $10 \times$ air $(0.13 \mathrm{NA})$ and $40 \times$ water immersion objectives (0.8NA) with infrared differential interference contrast (DIC) optics and an infrared CCD camera. Data were low-pass filtered at $6 \mathrm{kHz}$ and acquired at $10 \mathrm{kHz}$ with a Multiclamp 700B amplifier and a CV-7b headstage (Molecular Devices). Data were acquired using WaveSurfer (v0.953, Janelia Research Campus), and were analyzed using custom MATLAB scripts. Measurements were not adjusted for the liquid junction potential.

\section{Intrinsic excitability measurements}

To probe intrinsic excitability, whole cell recordings were performed on pyramidal neurons in current clamp in standard ACSF containing picrotoxin (PTX), 6,7-dinitroquinoxaline-2,3-dione (DNQX), and (2R)-amino-5-phosphonovaleric acid (APV) to block GABA, AMPA, and NMDA receptors, using a K-gluconate-based internal solution containing $100 \mathrm{~mm}$ K-gluconate, $10 \mathrm{~mm} \mathrm{KCl}, 10 \mathrm{~mm}$ HEPES, $5.37 \mathrm{~mm}$ biocytin, $10 \mathrm{~mm} \mathrm{Na}$-phosphocreatine, 4 $\mathrm{mm} \mathrm{Mg-ATP}$, and $0.3 \mathrm{~mm} \mathrm{Na-GTP}$, with sucrose added to bring osmolarity to $295 \mathrm{mOsm}$ and $\mathrm{KOH}$ added to bring $\mathrm{pH}$ to 7.35 in pipettes with resistance between 4 and 8 $\mathrm{m} \Omega$. To maintain resting membrane potential at $-70 \mathrm{mV}$, a small dc bias current was injected. One-second-long current injections of increasing amplitude, ranging from $-100 \mathrm{pA}$ to $400 \mathrm{pA}$ in intervals of $20 \mathrm{pA}$, were delivered to each neuron. Neurons were excluded from analysis if they displayed Rs $>25 \mathrm{M} \Omega, \mathrm{Vm}>-50 \mathrm{mV}$, or Rin $<80 \mathrm{M} \Omega$. Initial instantaneous firing rate was calculated by taking the reciprocal of the interspike interval between the first two action potentials. Mean instantaneous firing rate was calculated by averaging the reciprocal of the interspike intervals between all action potentials. Action potential threshold was determined by the membrane voltage when the derivative of the voltage first surpassed $20 \mathrm{~V} / \mathrm{s}$. Latency was defined as the time to first spike following current injection. Rheobase was defined as the injected current necessary to stimulate an action potential within $500 \mathrm{~ms}$. Action potential half-width was defined as the width of the action potential at the voltage halfway between the peak voltage and the threshold voltage.

\section{mEPSC recordings}

For spontaneous mEPSC recordings, neurons were voltage clamped to $-70 \mathrm{mV}$ in standard ACSF containing tetrodotoxin (TTX) $(0.2 \mu \mathrm{M})$, APV $(50 \mu \mathrm{M})$, and PTX (25 $\mu \mathrm{M})$ to block action potentials, NMDA receptors, and GABA receptors using a Cs-methanesufonate-based internal solution containing $115 \mathrm{~mm}$ Cs-methanesulfonate, 10 mM HEPES, 10 mm BAPTA.4Cs, $5.37 \mathrm{~mm}$ biocytin, $2 \mathrm{~mm}$ QX-314 Cl, $1.5 \mathrm{~mm} \mathrm{MgCl}$, $1 \mathrm{~mm}$ EGTA, $10 \mathrm{~mm} \mathrm{Na}_{2}-$ phosphocreatine, $4 \mathrm{~mm}$ ATP-Mg, and $0.3 \mathrm{~mm}$ GTP-Na, with sucrose added to bring osmolarity to $295 \mathrm{mOsm}$ and $\mathrm{CsOH}$ added to bring $\mathrm{pH}$ to 7.35 , in pipettes with resistance between 4 and $8 \mathrm{~m} \Omega$. $30 \mathrm{~s}$ traces were obtained every minute for $5 \mathrm{~min}$. mEPSC event inclusion criteria included rise times $<2 \mathrm{~ms}$ and amplitudes $>6 \mathrm{pA}$. Neurons were excluded from analysis if they displayed Rs $>20 \mathrm{M} \Omega, \mathrm{Vm}>-50 \mathrm{mV}$, or $\operatorname{Rin}<80 \mathrm{M} \Omega$.

\section{Immunohistochemistry}

Following transcardial perfusion with $4 \%$ paraformaldehyde (PFA) in $0.01 \mathrm{~m}$ PBS, brains were postfixed overnight in chilled perfusion solution before being transferred to $0.01 \mathrm{~m}$ PBS. $50-\mu \mathrm{m}$ coronal slices were cut the following day on a vibratome, then washed three times and stored in $0.01 \mathrm{~m}$ PBS with $0.05 \% \mathrm{NaN}_{3}$ at $4^{\circ} \mathrm{C}$ until staining.

Slices were rinsed three times in $0.01 \mathrm{~m}$ PBS, then incubated in the primary antibody solution overnight $(0.3 \%$ Triton $\mathrm{X}-100,0.05 \% \mathrm{NaN}_{3}, 1 \% \mathrm{BSA}$, and primary antibody in $0.01 \mathrm{M}$ PBS). The following day, slices were rinsed three times in $0.01 \mathrm{~m}$ PBS, then incubated in the secondary antibody solution for $2 \mathrm{~h}(1 \% \mathrm{BSA}$ and secondary antibody in $0.01 \mathrm{M}$ PBS). Slices were then mounted on a glass slide with an anti-fade medium (Fluoromount-G), coverslipped, and allowed to dry overnight. Images were taken on a Leica SP5 confocal microscope. Image stacks with 488-, 543-, and 647-nm lasers were obtained using a $20 \times$ objective with an optical section height (z-step) of $\sim 2 \mu \mathrm{m}$. Images were subsequently analyzed in ImageJ.

We used the following antibodies:

anti-CaMKIV (1:1000, Santa Cruz Biotechnology sc136249, RRID:AB_2275109),

anti-CaMKIV (1:1000, Sigma C2851, RRID:AB_258821), anti-CaMKIV (1:1000, BD Biosciences 610275, RRID: AB_397670),

anti-pCaMKIV (1:200, Santa Cruz Biotechnology sc28443-R, RRID:AB_2068399),

anti-VGlut2 (1:10000, Millipore AB2251, RRID:AB 1587626), 
A
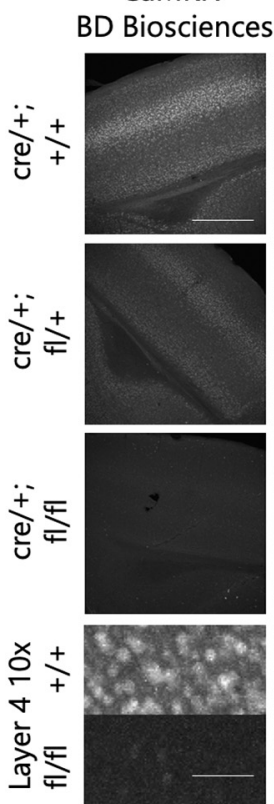

CaMKIV
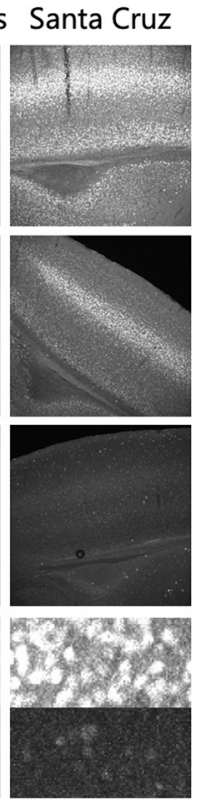

CaMKIV Sigma Aldrich
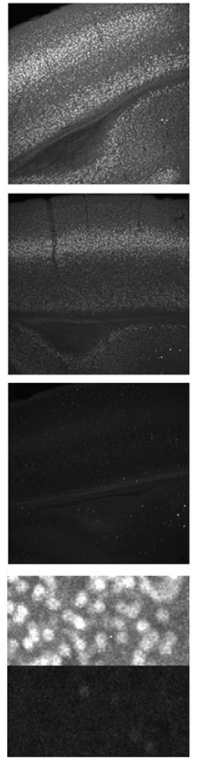

pCaMKIV B
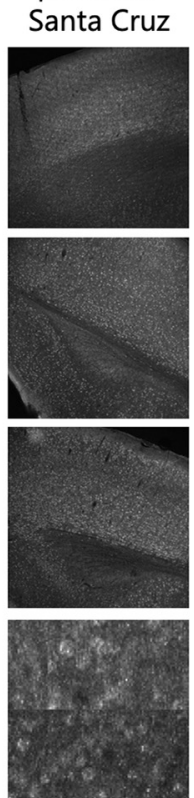

D
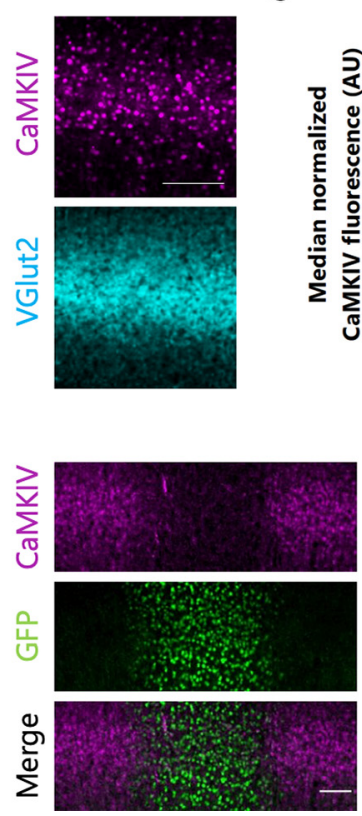

C

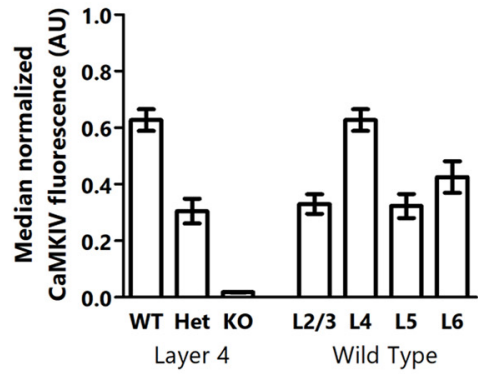

E

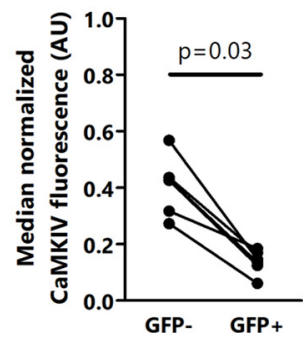

Figure 1. CaMKIV is expressed in excitatory neurons, and at higher levels in L4 of mouse V1. A, anti-CaMKIV and anti-phosphoCaMKIV staining in mouse V1. Each column represents a different antibody, and each row represents a different genotype. Scale bars: $500 \mu \mathrm{m}$ (top three rows) and $50 \mu \mathrm{m}$ (bottom row). B, Expression of CaMKIV and VGlut2. Scale bar: $100 \mu \mathrm{m}$. C, left, Quantification of CaMKIV expression in mice with 2, 1, or 0 copies of CaMKIV. Right, Quantification of CaMKIV expression in different layers in mice with two copies of CaMKIV. $\boldsymbol{D}$, Immunofluorescence of CaMKIV and GFP $7 \mathrm{~d}$ after Cre-GFP injection into floxCaMKIV mice. E, Quantification of CaMKIV expression in GFP-negative (GFP-) and GFP-positive (GFP+) neurons $7 \mathrm{~d}$ after Cre-GFP injection into flox-CaMKIV mice; $p$ value calculated by Wilcoxon matched pairs test.

Goat anti-Mouse IgG Alexa Fluor 555 (1:300, Thermo Fisher A-21424, RRID:AB_141780),

Goat anti-Rabbit IgG Alexa Fluor 488 (1:300, Thermo Fisher A-11034, RRID:AB_2576217),

Goat anti-Guinea Pig IgG Alexa Fluor 647 (1:300, Thermo Fisher A-21450, RRID:AB_2735091).

When using the polyclonal phospho-CaMKIV (T196) antibody (sc-28443-R, Santa Cruz Biotechnology), we did not observe any difference in staining between wild-type and conditional CaMKIV knock-down mice. This suggests that the Santa Cruz Biotechnology sc-28443-R antibody, when used for immunostaining, is not specific for pCaMKIV. We attempted to immunolabel phosphoCaMKIV with antibodies obtained from multiple other sources with no success.

\section{Statistical analysis}

Data analysis was performed using custom MATLAB scripts or GraphPad Prism. The number of animals and neurons for each experiment, as well as statistical tests used, are stated in each figure legend. Individual data points indicate measurements from one neuron. Horizontal bars and connected points (in F-I curves) represent means, error bars represent SEM unless otherwise noted. Data were tested for normality using a Shapiro-Wilk normality test, and compared using a $t$ test (for normally distributed data), Mann-Whitney $U$ test (non-normally distributed data), or two-way ANOVA (series data).

\section{Results}

\section{CaMKIV is expressed in excitatory neurons in mouse V1}

CaMKIV is a $\mathrm{Ca}^{2+}$-dependent kinase with many targets, including the transcription factor CREB and the splicing factor Nova-2 (Cruzalegui and Means, 1993; Lonze and Ginty, 2002; Li et al., 2020), that has been implicated in synaptic scaling and plasticity of intrinsic excitability in neocortical pyramidal neurons in vitro (Joseph and Turrigiano, 2017). However, its role in these processes in vivo has not yet been tested.

Before manipulating CaMKIV signaling, we first determined its expression pattern in mouse visual cortex. Using three different primary antibodies, we found that CaMKIV is expressed in a layer-specific manner in mouse visual cortex. Consistent with published RNA-seq data (Hrvatin et al., 2018), we found robust CaMKIV expression in all layers of $\mathrm{V} 1$, with highest expression levels in L4 as indicated by its similar expression pattern to that of VGlut2, which labels the thalamocortical afferents that project to L4 (Fig. 1A, top row, B,C). To confirm the specificity of each of these antibodies, and to identify the neuronal types that express CaMKIV in visual cortex in vivo, we selectively removed CaMKIV from pyramidal neurons by crossing a flox-CaMKIV mouse (Casanova et al., 2002) with an EMX1-Cre mouse, which expresses Cre exclusively in pyramidal neurons (Gorski et al., 2002). In mice in which both copies of the CaMKIV gene were constitutively removed in pyramidal neurons, we saw a dramatic 
A

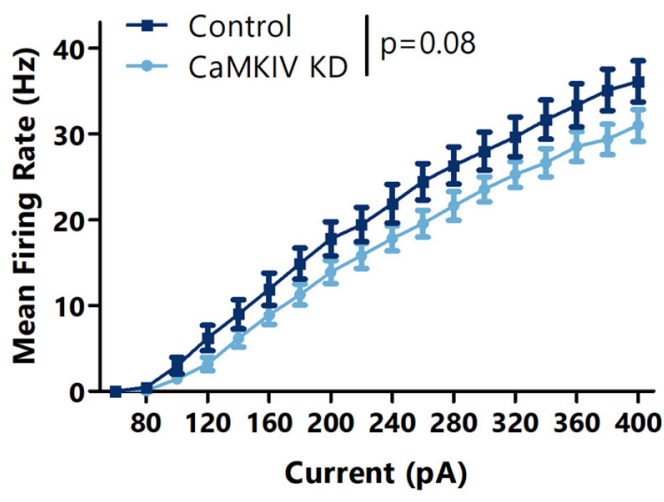

B

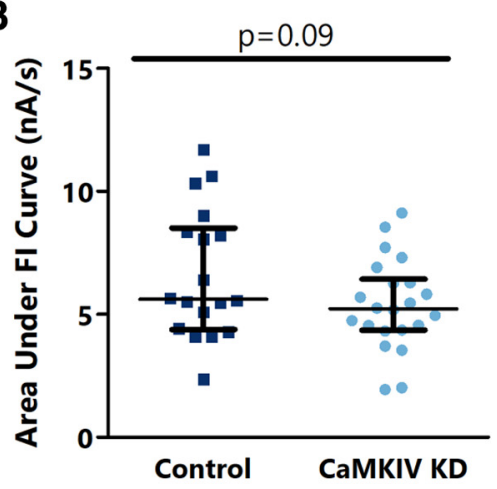

E

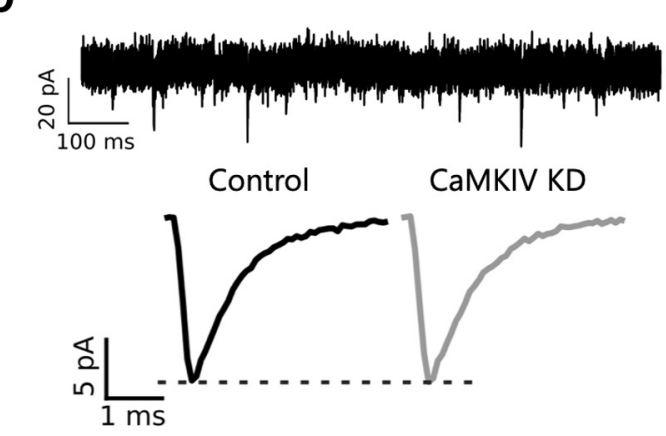

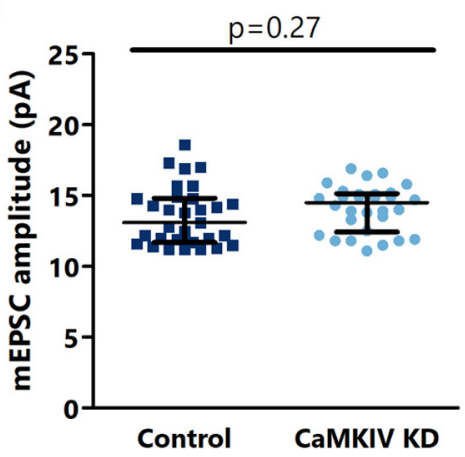

C

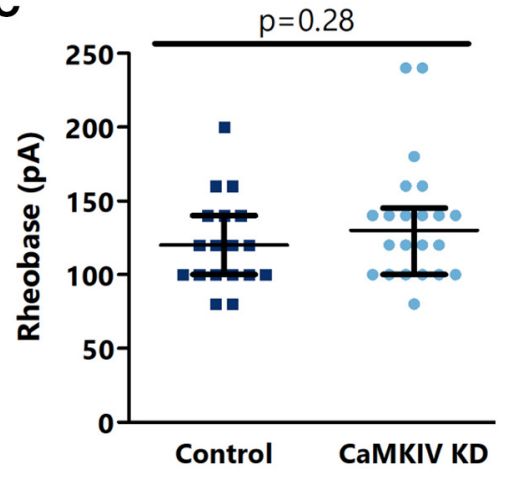

Figure 2. CaMKIV signaling does not negatively regulate intrinsic excitability or mEPSC amplitude or frequency in L2/3 pyramidal neurons in V1m. $\boldsymbol{A}$, Firing rate-current $(\mathrm{F}-\mathrm{l})$ curves for L2/3 control and AAV-mediated CaMKIV knock-down neurons. $\boldsymbol{B}$, Quantification of the area under the F-I curves from $\boldsymbol{A}$. C, Comparison of rheobase current between L2/3 control and CaMKIV knock-down neurons. $\boldsymbol{D}$, top, Representative mEPSC trace from L2/3 control neuron. Bottom, Average mEPSC traces for L2/3 control and CaMKIV knock-down neurons. $\boldsymbol{E}$, Comparison of mEPSC amplitude between L2/3 control and CaMKIV knock-down neurons. $\boldsymbol{F}$, Comparison of mEPSC frequency between L2/3 control and CaMKIV knock-down neurons. $\boldsymbol{A}-\boldsymbol{C}, n=18-22$ neurons from 3 animals per condition. $\boldsymbol{D}-\boldsymbol{F}, n=30-31$ neurons from 4 animals per condition; $p$ values calculated by two-way ANOVA in $\boldsymbol{A}, t$ test in $\boldsymbol{B}, \boldsymbol{E}$, and Mann-Whitney $U$ test in $\boldsymbol{C}, \boldsymbol{F}$. For details, see statistical table (Table 1).

decrease in CaMKIV expression in all layers regardless of which antibody we used (Fig. 1A). We also looked at CaMKIV expression in animals with only one copy of the CaMKIV gene present in excitatory neurons, and found that CaMKIV levels were decreased by about half (Fig. $1 A)$. This indicates that these antibodies are specific for CaMKIV and that CaMKIV is primarily expressed in excitatory neurons, consistent with its expression pattern in vitro (Cohen et al., 2016; Ma et al., 2014). In contrast, with a commonly used polyclonal phospho-CaMKIV (T196) antibody (sc-28443-R, Santa Cruz Biotechnology) we detected no qualitative difference in labeling between the mice with two copies of the CaMKIV gene and those with zero, arguing that this phospho-CaMKIV antibody is not specific for phospho-CaMKIV (Fig. 1A). Attempts to use other phospho-CaMKIV antibodies on tissue slices were unsuccessful.

\section{CaMKIV signaling in vivo is not required for the maintenance of intrinsic excitability or mEPSC amplitude or frequency}

Next, we sought to test whether in vivo CaMKIV signaling is required for the maintenance of basal excitatory synaptic properties or intrinsic excitability during the rodent visual system critical period. In cultured hippocampal neurons, CaMKIV protein level is rate-limiting in the pathway that links membrane depolarization to CREB phosphorylation, suggesting that altering CaMKIV protein levels should trigger a homeostatic response that can be revealed by measuring basal synaptic and intrinsic properties (Cohen et al., 2016). To avoid compensation that might be arise following constitutive CaMKIV knock-out, we used a viral approach to reduce CaMKIV protein levels with temporal and spatial specificity. We found that $7 \mathrm{~d}$ after we injected a Cre-GFP virus (driven by the CaMKII promotor fragment to restrict expression to excitatory neurons) into mouse visual cortex, we saw a robust decrease in CaMKIV protein levels (median decrease of $70 \%$; Fig. 1D,E).

Since robust synaptic scaling and plasticity of intrinsic excitability have both been observed in L2/3 pyramidal neurons during the critical period in $\mathrm{V} 1 \mathrm{~m}$ (Lambo and Turrigiano, 2013), we injected our Cre-GFP into V1m at P15 and measured the intrinsic excitability and mEPSC amplitude and frequency in pyramidal neurons in infected and uninfected control hemispheres one week later. In contrast to the increase in intrinsic excitability and 
Table 1: Statistical table

\begin{tabular}{|c|c|c|c|c|}
\hline & Structure & Statistical test & $p$ value & $\mathrm{Cl} /$ ranks \\
\hline \multicolumn{5}{|l|}{ Figure 1} \\
\hline $\mathrm{e}$ & Not normal & Wilcoxon matched pairs & 0.0313 & 21,0 \\
\hline \multicolumn{5}{|l|}{ Figure 2} \\
\hline a & Series data & Two-way RM ANOVA & 0.0846 & \\
\hline $\mathrm{b}$ & Normal & Unpaired $t$ test & 0.085 & $6.623 \pm 0.6184 N=18,5.374 \pm 0.3916 N=22$ \\
\hline c & Not normal & Mann-Whitney $U$ test & 0.2836 & 330,490 \\
\hline e & Normal & Unpaired $t$ test & 0.2694 & $13.55 \pm 0.3736 N=31,14.09 \pm 0.2983 N=30$ \\
\hline f & Not normal & Mann-Whitney $U$ test & 0.3633 & $897.5,993.5$ \\
\hline \multicolumn{5}{|l|}{ Figure 3} \\
\hline a & Series data & Two-way RM ANOVA & 0.085 & \\
\hline b & Normal & Unpaired $t$ test & 0.5139 & $10.47 \pm 0.5924 N=27,9.922 \pm 0.5781 N=30$ \\
\hline c & Not normal & Mann-Whitney $U$ test & 0.779 & $765.5,887.5$ \\
\hline e & Normal & Unpaired $t$ test & 0.7955 & $14.53 \pm 0.3348 N=31,14.65 \pm 0.3131 N=31$ \\
\hline f & Normal & Unpaired $t$ test & 0.1119 & $9.974 \pm 0.5735 N=31,11.25 \pm 0.5429 N=31$ \\
\hline \multicolumn{5}{|r|}{ - } \\
\hline a & Series data & Two-way RM ANOVA & 0.3969 & \\
\hline b & Not normal & Mann-Whitney $U$ test & 0.506 & 394,467 \\
\hline c & Not normal & Mann-Whitney $U$ test & 0.7259 & $433.5,427.5$ \\
\hline e & Not normal & Mann-Whitney $U$ test & 0.315 & $13.95 \pm 0.6251 N=17,12.99 \pm 0.7150 N=17$ \\
\hline f & Not normal & Mann-Whitney $U$ test & 0.2658 & $5.717 \pm 0.5063 N=17,6.576 \pm 0.5653 N=17$ \\
\hline \multicolumn{5}{|r|}{ 由. } \\
\hline a & Series data & Two-way RM ANOVA & 0.1488 & \\
\hline a & Series data & Two-way RM ANOVA & 0.5697 & \\
\hline$b$ & Normal & Unpaired $t$ test & 0.1400 & $5.047 \pm 0.3205 N=24,5.771 \pm 0.3480 N=17$ \\
\hline b & Normal & Unpaired $t$ test & 0.5630 & $4.555 \pm 0.4194 N=19,4.903 \pm 0.4186 N=23$ \\
\hline c & Normal & Unpaired $t$ test & 0.0533 & $156.7 \pm 8.982 N=24,131.8 \pm 7.681 N=17$ \\
\hline c & Normal & Unpaired $t$ test & 0.2457 & $162.1 \pm 7.631 N=19,151.3 \pm 5.456 N=23$ \\
\hline d & Series data & Two-way RM ANOVA & 0.006 & \\
\hline d & Series data & Two-way RM ANOVA & 0.0469 & \\
\hline e & Normal & Unpaired $t$ test & 0.0059 & $3.974 \pm 0.2320 N=21,5.314 \pm 0.3984 N=21$ \\
\hline e & Normal & Unpaired $t$ test & 0.0442 & $4.063 \pm 0.3160 N=14,5.430 \pm 0.4976 N=20$ \\
\hline f & Not normal & Mann-Whitney $U$ test & 0.0072 & 556,347 \\
\hline f & Normal & Unpaired $t$ test & 0.0227 & $168.6 \pm 7.764 N=14,142.0 \pm 7.525 N=20$ \\
\hline i & Not normal & Mann-Whitney $U$ test & 0.7248 & 455,406 \\
\hline i & Normal & Unpaired $t$ test & 0.8161 & $0.9152 \pm 0.03274 N=14,0.9050 \pm 0.02835 N=20$ \\
\hline \multicolumn{5}{|r|}{ 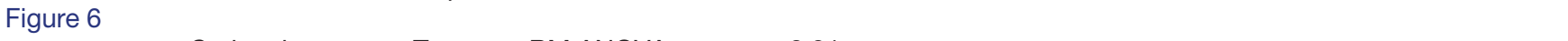 } \\
\hline a & Series data & Two-way RM ANOVA & 0.21 & \\
\hline b & Series data & Two-way RM ANOVA & $<0.0001$ & \\
\hline c & Series data & Two-way RM ANOVA & 0.662 & \\
\hline c & Series data & Two-way RM ANOVA & $<0.0001$ & \\
\hline$d$ & Not normal & Mann-Whitney $U$ test & 0.7443 & 407,454 \\
\hline d & Normal & Unpaired $t$ test & $<0.0001$ & $2.807 \pm 0.09702 N=27,6.091 \pm 0.4462 N=23$ \\
\hline e & Normal & Unpaired $t$ test & 0.4124 & $-33.44 \pm 0.5506 N=20,-32.67 \pm 0.7326 N=21$ \\
\hline e & Not normal & Mann-Whitney $U$ test & $<0.0001$ & 378,897 \\
\hline f & Not normal & Mann-Whitney $U$ test & 0.5929 & 441,420 \\
\hline f & Not normal & Mann-Whitney $U$ test & $<0.0001$ & 920,355 \\
\hline $\mathrm{h}$ & Not normal & Mann-Whitney $U$ test & 0.5401 & 361,269 \\
\hline i & Normal & Unpaired $t$ test & 0.3617 & $19.53 \pm 1.682 N=19,17.24 \pm 1.803 N=16$ \\
\hline
\end{tabular}

Boldface indicates $p<0.05$

mEPSC amplitude seen in cultured pyramidal neurons following dnCaMKIV expression (Joseph and Turrigiano, 2017), we saw a small but not statistically significant decrease in intrinsic excitability in neurons lacking CaMKIV (Fig. 2A-C), and no change in either mEPSC amplitude or frequency (Fig. 2D-F).

To test whether the lack of an effect of decreasing CaMKIV protein levels was layer specific, we next knocked out CaMKIV in L4, where its expression is highest. As in L2/3, we saw a small but not statistically significant decrease in intrinsic excitability following CaMKIV knock-down instead of the expected increase (Fig. $3 A-C$ ), and no difference in mEPSC amplitude or frequency (Fig. 3D-F).

Since the in vitro data were obtained using dnCaMKIV rather than CaMKIV knock-down, we tested whether our results were because of an unanticipated difference in the effects of CaMKIV knock-down and dnCaMKIV. However, consistent with the our CaMKIV knock-down data, we found that dnCaMKIV expression did not cause an increase 
A

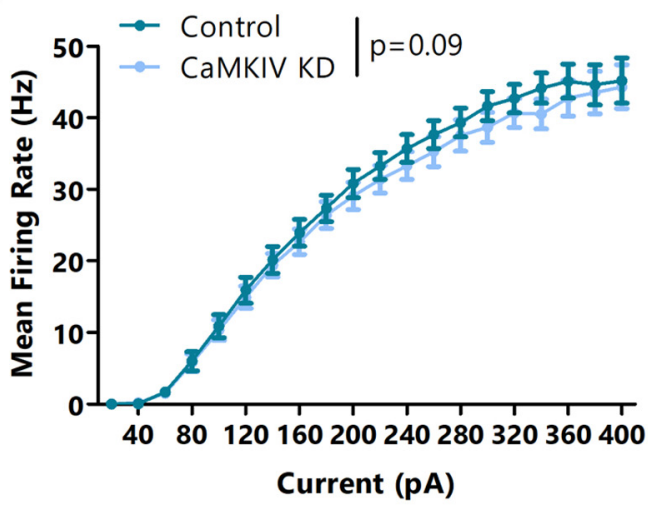

D

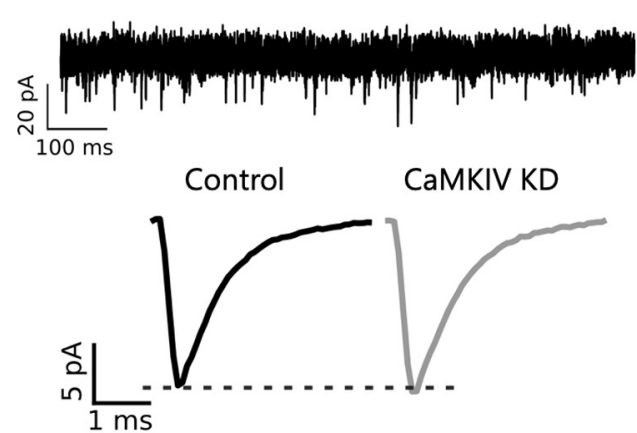

B

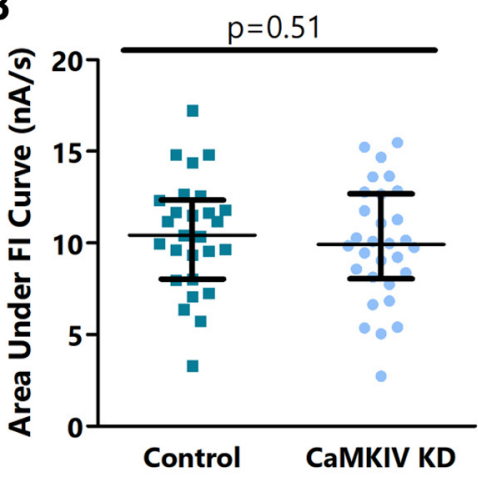

E

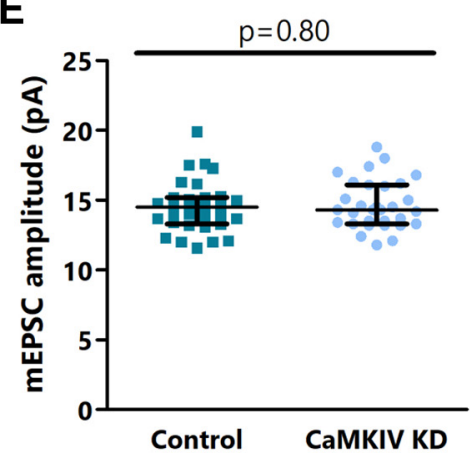

C

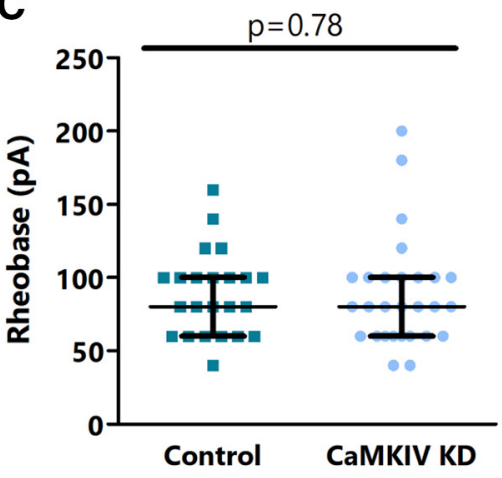

$\mathbf{F}$

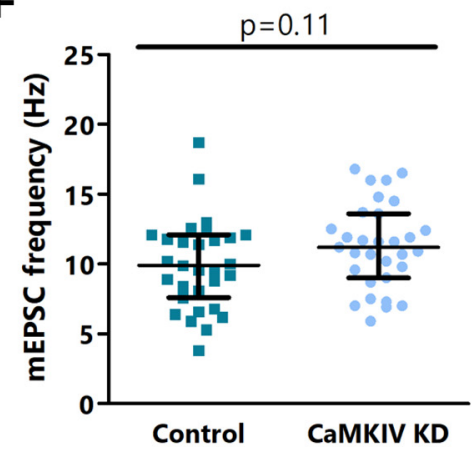

Figure 3. CaMKIV signaling does not negatively regulate intrinsic excitability or mEPSC amplitude or frequency in L4 pyramidal neurons in V1m. A, Firing rate-current (F-l) curves for L4 control and AAV-mediated CaMKIV knock-down neurons. B, Quantification of the area under the F-I curves from $\boldsymbol{A}$. C , Comparison of rheobase current between L4 control and CaMKIV knock-down neurons. $\boldsymbol{D}$, top, Representative mEPSC trace from L4 control neuron. Bottom, Average mEPSC traces for L4 control and CaMKIV knock-down neurons. $\boldsymbol{E}$, Comparison of mEPSC amplitude between L4 control and CaMKIV knock-down neurons. $\boldsymbol{F}$, Comparison of mEPSC frequency between L4 control and CaMKIV knock-down neurons. $\boldsymbol{A}-\boldsymbol{C}, n=27-30$ neurons from 4 animals per condition. $\boldsymbol{D}-\boldsymbol{F}, n=31$ neurons from 4 animals per condition; $p$ values calculated by two-way ANOVA in $\boldsymbol{A}, t$ test in $\boldsymbol{B}, \boldsymbol{E}, \boldsymbol{F}$, and Mann-Whitney $U$ test in $\boldsymbol{C}$. For details, see statistical table (Table 1).

in intrinsic excitability (Fig. $4 A-C$ ) or mEPSC amplitude or frequency (Fig. $4 D-F$ ). Taken together, our data show that in vivo CaMKIV activity is not required for maintaining baseline excitability and mEPSC amplitude in L2/3 or L4 pyramidal neurons.

\section{CaMKIV signaling is not required for plasticity of intrinsic excitability in V1m or V1b}

Although CaMKIV is not required for regulating baseline synaptic and intrinsic properties, it is possible that it is important for homeostatic plasticity in response to persistent changes in input. The intrinsic excitability of excitatory neurons in V1b increases following $6 \mathrm{~d}$ of MD (Lambo and Turrigiano, 2013; Moore et al., 2018), but it is not known which molecular pathways regulate this change in intrinsic excitability. The effects of $6 \mathrm{~d}$ of MD on intrinsic excitability in $\mathrm{V} 1 \mathrm{~m}$ are also unknown. To determine whether CaMKIV signaling is required for plasticity of intrinsic excitability we knocked out CaMKIV in V1m or V1b in flox-CaMKIV mice, then sutured one eyelid shut just before the beginning of the critical period (P21) and measured intrinsic excitability $6 \mathrm{~d}$ later (Lambo and Turrigiano, 2013).
In $\mathrm{V} 1 \mathrm{~m}$, we found that $6 \mathrm{~d}$ of $\mathrm{MD}$ did not cause a change in intrinsic excitability (Fig. $5 A-C$ ). As before (Fig. $2 A-C$ ), we found that CaMKIV knock-down caused a slight decrease in intrinsic excitability, but the decrease in CaMKIV levels did not alter the lack of effect of $6 \mathrm{~d}$ of MD on intrinsic excitability. We observed an increased intrinsic excitability in $\mathrm{V} 1 \mathrm{~b}$ control animals following $6 \mathrm{~d}$ of $\mathrm{MD}$, as expected, but this plasticity was also not affected by CaMKIV knock-down (Fig. 5D-F). Thus, in vivo CaMKIV signaling is not required for the regulation of intrinsic excitability in L2/3 pyramidal neurons in response to MD.

CaMKIV signaling has also recently been implicated in the homeostatic regulation of the width of rebound action potentials that can follow prolonged hyperpolarization in vitro (Li et al., 2020). However, its role in regulating the width of action potentials produced by direct depolarization has never been tested. To determine whether action potential width is homeostatically regulated in pyramidal neurons in visual cortex, we measured the width of action potentials following rheobase current injection in V1b in our sham and monocularly deprived animals described above. We found that although $6 \mathrm{~d}$ of MD caused a clear increase in intrinsic 
A

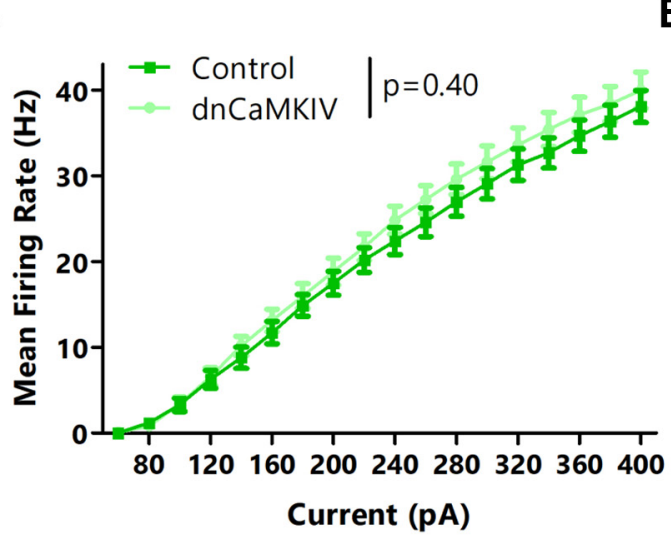

B

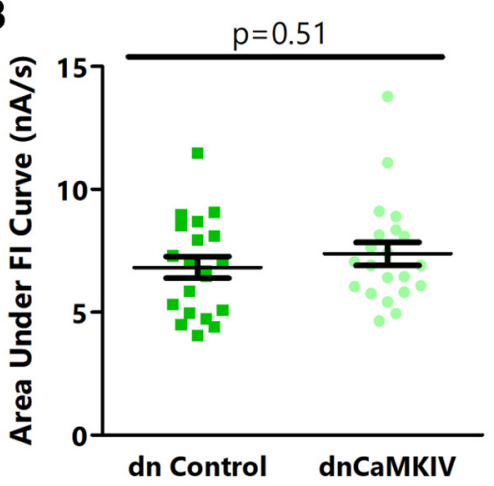

E

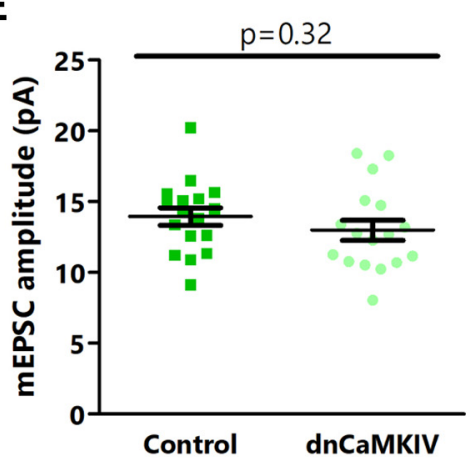

C

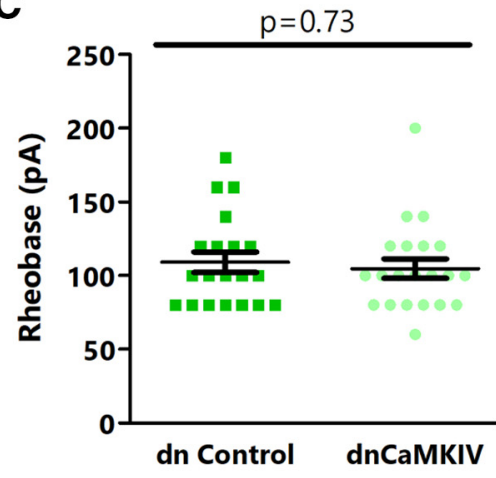

D

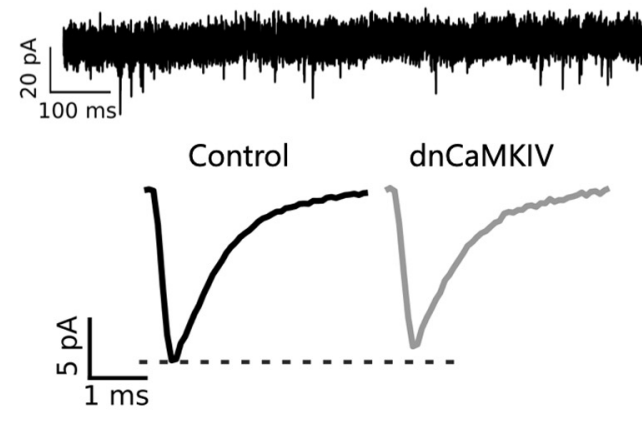

Figure 4. dnCaMKIV signaling does not negatively regulate intrinsic excitability, or mEPSC amplitude or frequency in L2/3 pyramidal neurons in V1m. $\boldsymbol{A}$, Firing rate-current (F-I) curves for L2/3 control and AAV-mediated dnCaMKIV-expressing neurons. $\boldsymbol{B}$, Quantification of the area under the F-I curves from $\boldsymbol{A}$. C, Comparison of rheobase current between L2/3 control and dnCaMKIV. $\boldsymbol{D}$, top, Representative mEPSC trace from L2/3 control neuron. Bottom, Average mEPSC traces for L2/3 control and dnCaMKIV neurons. $\boldsymbol{E}$, Comparison of mEPSC amplitude between L2/3 control and dnCaMKIV neurons. $\boldsymbol{F}$, Comparison of mEPSC frequency between $\mathrm{L} 2 / 3$ control and dnCaMKIV neurons. $\boldsymbol{A}-\boldsymbol{C}, n=17$ neurons from 3 animals per condition. $\boldsymbol{D}-\boldsymbol{F}, n=17$ neurons from 3 animals per condition; $p$ values calculated by two-way ANOVA in $\boldsymbol{A}, t$ test in $\boldsymbol{E}, \boldsymbol{F}$, and Mann-Whitney $U$ test in $\boldsymbol{B}, \boldsymbol{C}$. For details, see statistical table (Table 1).

excitability in $\mathrm{V} 1 \mathrm{~b}$, it did not affect action potential width (Fig. 5G,I). CaMKIV knock-down also failed to alter the action potential width in sham or deprived mice (Fig. 5H,l). Together, these results demonstrate that as with intrinsic excitability, in vivo CaMKIV signaling does not regulate action potential width in pyramidal neurons in visual cortex.

\section{Constitutive activation of CaMKIV causes complex changes in intrinsic properties}

Our data show that in vivo CaMKIV signaling is dispensable for homeostatic regulation of neuronal excitability in rodent visual cortex. However, they do not rule out the possibility that in vivo CaMKIV signaling does influence mEPSC amplitude or intrinsic signaling excitability, but its loss is robustly compensated. Acute expression of caCaMKIV in pyramidal neurons in rodent hippocampus induces silent synapses, which can be detected as to an increase in mEPSC frequency, despite the lack of effect of dnCaMKIV on hippocampal mEPSCs (Marie et al., 2005). To determine whether a similar phenomenon occurs in pyramidal neurons in rodent visual cortex, we virally expressed caCaMKIV in vivo and measured intrinsic and synaptic properties.
In stark contrast to our observations following dnCaMKIV expression, we found that caCaMKIV, in which the autoinhibitory domain is inactivated (Wayman et al., 2011), caused robust and complex changes in intrinsic excitability. Neurons expressing caCaMKIV showed decreased excitability in response to small amplitude current injections, but with larger current injections their excitability was increased (Fig. 6A). Neurons expressing caCaMKIV also showed a lower initial instantaneous firing rate (Fig. 6B), less spike frequency adaptation during the spike train (Fig. 6C), tended to fire with a delay following depolarization (Fig. $6 D$ ), and had a higher action potential threshold than control neurons (Fig. $6 E)$. They did not show any differences in input resistance, but had an increased resting membrane potential and decreased capacitance (Fig. 6F). In contrast, none of these parameters differed between dnCaMKIV and control neurons (Fig. 6C-F). These myriad differences following caCaMKIV expression suggest a complex rebalancing of ion channel expression or conductance as a result of constitutive kinase activity.

Finally, we tested whether caCaMKIV also affects synaptic properties by measuring mEPSC amplitude and frequency. As with the CaMKIV knock-down and dnCaMKIV, and in contrast to previous reports from hippocampal 
A

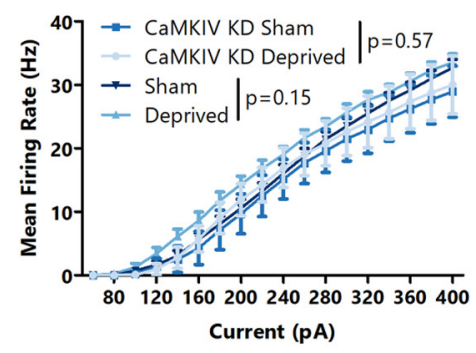

D

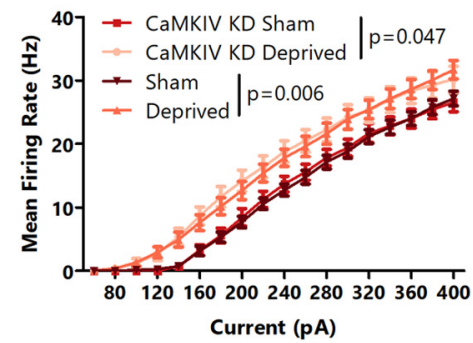

G

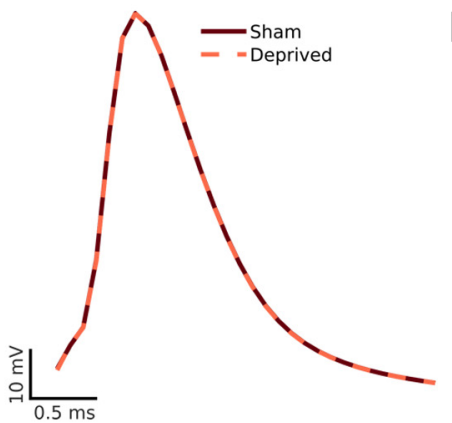

B

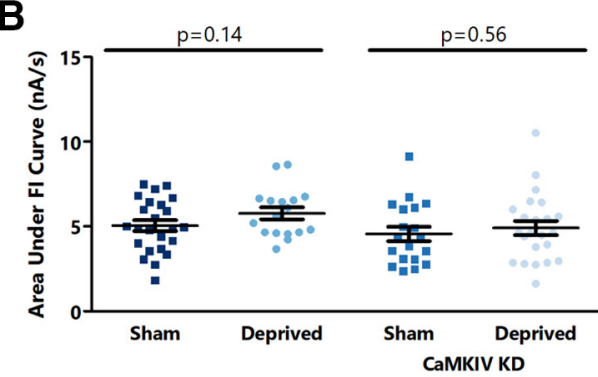

E

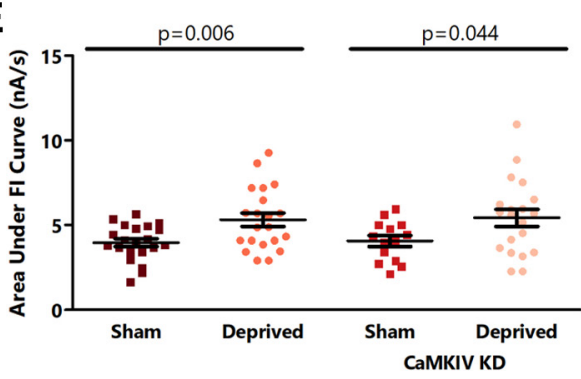

H

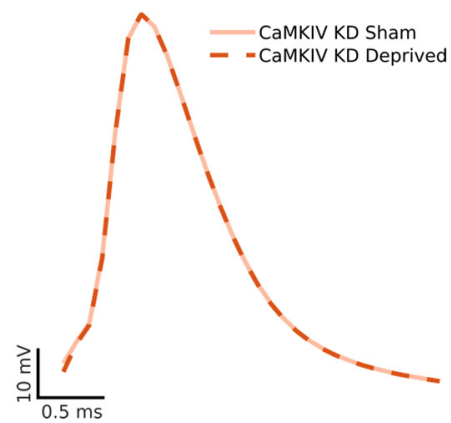

C

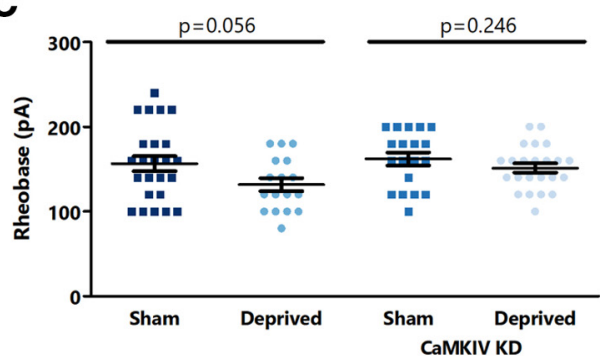

$\mathbf{F}$

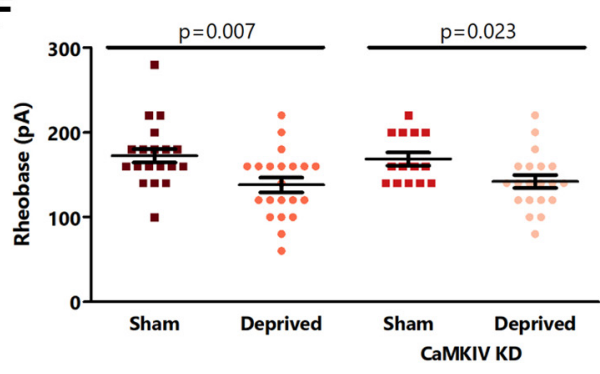

Figure 5. CaMKIV is not required for plasticity of intrinsic excitability in V1m or V1b following a 6-d MD. $\boldsymbol{A}$, Firing rate-current (F-I) curves for L2/3 sham and monocularly deprived control and CaMKIV knock-down neurons in V1m. $\boldsymbol{B}$, Quantification of the area under the $\mathrm{F}-\mathrm{I}$ curves from $\boldsymbol{A}$. C, Comparison of rheobase current between L2/3 sham and monocularly deprived control and CaMKIV knock-down neurons in V1m. $\boldsymbol{D}$, Firing rate-current $(F-l)$ curves for $\mathrm{L} 2 / 3$ sham and monocularly deprived control and CaMKIV knock-down neurons in V1b. $\boldsymbol{E}$, Quantification of the area under the F-I curves from $\boldsymbol{D}$. $\boldsymbol{F}$, Comparison of rheobase current between L2/3 sham and monocularly deprived control and CaMKIV knock-down neurons in V1b. G, Average action potential waveforms for the first action potential measured at rheobase for sham and monocularly deprived neurons in V1b. $\boldsymbol{H}$, Average action potential waveforms for the first action potential measured at rheobase for sham and monocularly deprived CaMKIV knock-down neurons in V1b. I, Action potential half width at rheobase for sham and monocularly deprived control and CaMKIV knock-down neurons in V1b. All comparisons are to mice of the same genotype that were reared and treated identically, except eyelids were not sutured; $\boldsymbol{A}-\boldsymbol{C}$. $n=17-24$ neurons from 3 animals per condition. $\boldsymbol{D}-\boldsymbol{I}$ : $n=18$ neurons from 3 animals per condition. $p$ values calculated by two-way ANOVA in $\boldsymbol{A}, \boldsymbol{D}, \boldsymbol{t}$-test in $\boldsymbol{B}, \boldsymbol{C}, \boldsymbol{E}, \boldsymbol{F}, \boldsymbol{I}$, and Mann-Whitney $\boldsymbol{U}$-test in $\boldsymbol{E}, \boldsymbol{F}, \boldsymbol{I}$. For details, see statistical table (Table 1 ).

pyramidal neurons (Marie et al., 2005), we saw no difference in mEPSC amplitude or frequency between caCaMKIV neurons and control neurons (Fig. 6G-I). Taken together, these results demonstrate that constitutive CaMKIV signaling in vivo can alter neuronal excitability but not mEPSC frequency or amplitude in cortical pyramidal neurons, but do not support a clear role for CaMKIV signaling in regulating baseline neuronal excitability.

\section{Discussion}

Multiple forms of homeostatic plasticity, most notably synaptic scaling and plasticity of intrinsic excitability, stabilize firing rates of cortical neurons within target ranges.
In immature cultured cortical neurons, CaMKIV signaling modulates baseline firing rate by jointly regulating excitatory synaptic strength and intrinsic excitability (Joseph and Turrigiano, 2017), suggesting that it may serve as a master regulator of different forms of homeostatic plasticity. Here, we tested whether in vivo CaMKIV signaling similarly regulates baseline synaptic properties and intrinsic excitability in rodent visual cortex, at a time point (the visual system critical period) when neuronal properties and circuits are highly plastic. We found that neither conditional CaMKIV knock-down nor dnCaMKIV mimicked the increase in mEPSC amplitude and intrinsic excitability seen with dnCaMKIV expression in cultured neurons. Similarly, CaMKIV signaling was not necessary 
A

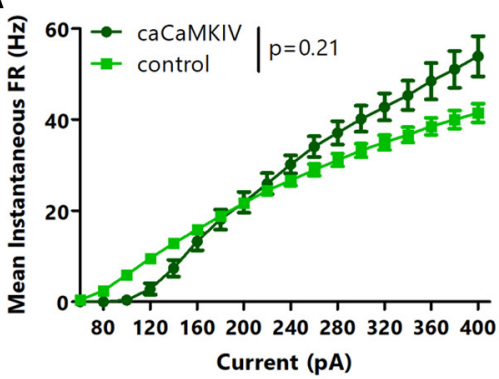

D

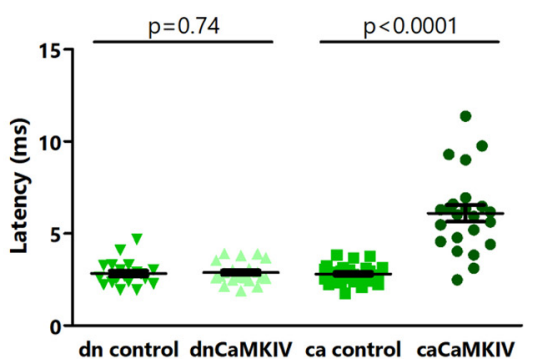

G

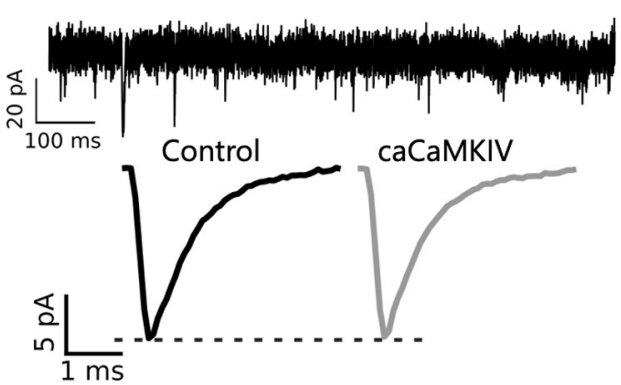

B

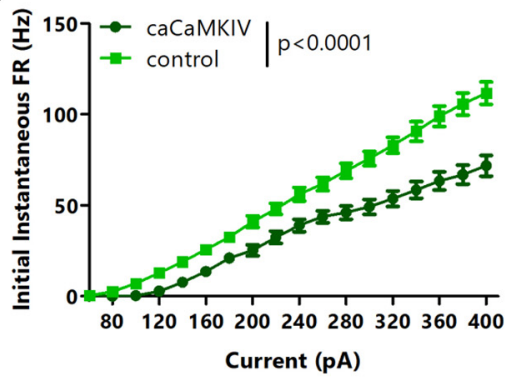

E

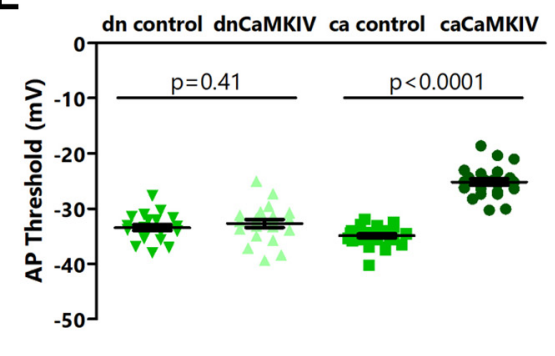

H

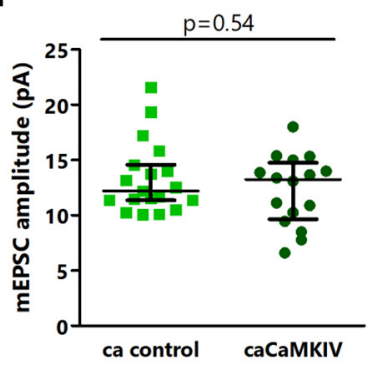

C

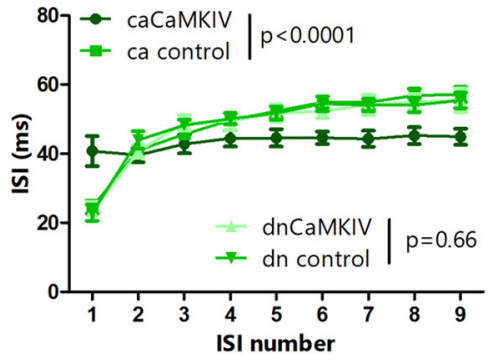

$\mathbf{F}$

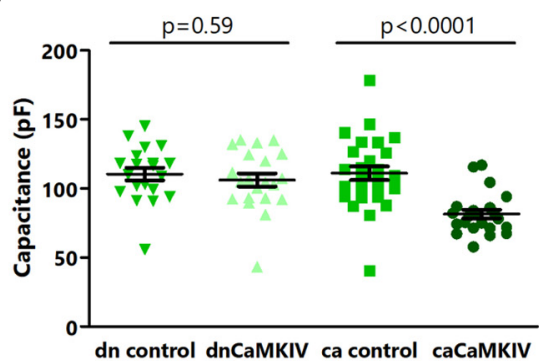

I

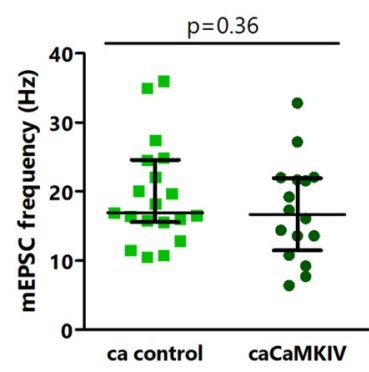

Figure 6. caCaMKIV disrupts intrinsic excitability but does not affect mEPSC amplitude or frequency. $\boldsymbol{A}$, Mean instantaneous firing rate-current $(\mathrm{F}-\mathrm{I})$ curves for $\mathrm{L} 2 / 3$ control and AAV-mediated caCaMKIV-expressing neurons. $\boldsymbol{B}$, Initial instantaneous firing rate-current $(F-I)$ curves for $L 2 / 3$ control and AAV-mediated caCaMKIV-expressing neurons. $\boldsymbol{C}$, Comparison of interspike interval versus spike number for caCaMKIV and dnCaMKIV neurons. $\boldsymbol{D}$, Comparison of latency to first action potential for caCaMKIV and dnCaMKIV neurons. $\boldsymbol{E}$, Comparison of action potential threshold for caCaMKIV and dnCaMKIV neurons. $\boldsymbol{F}$, Comparison of membrane capacitance for caCaMKIV and dnCaMKIV neurons. G, top, Representative mEPSC trace from L2/3 control neuron. Bottom, Average mEPSC traces for L2/3 control and caCaMKIV neurons. $\boldsymbol{H}$, Comparison of mEPSC amplitude between L2/3 control and caCaMKIV neurons. I, Comparison of mEPSC frequency between $\mathrm{L} 2 / 3$ control and caCaMKIV neurons. $\boldsymbol{A}-\boldsymbol{F}, n=18-27$ neurons from 3 animals per condition. $\mathbf{G}-\boldsymbol{I}, n=16-19$ neurons from 4 animals per condition; $p$ values calculated by two-way ANOVA in $\boldsymbol{A}-\boldsymbol{C}$, $t$ test in $\boldsymbol{D}, \boldsymbol{E}, \boldsymbol{I}$, and Mann-Whitney $U$ test in $\boldsymbol{D}, \boldsymbol{E}, \boldsymbol{F}, \boldsymbol{H}$. For details, see statistical table (Table 1).

for the increase in intrinsic excitability seen in $\mathrm{V} 1 \mathrm{~b}$ following 6-d MD (Lambo and Turrigiano, 2013; Moore et al., 2018), and neither MD nor CaMKIV knock-down caused homeostatic regulation of action potential width. Expression of caCaMKIV caused a number of changes in intrinsic properties but did not affect mEPSC amplitude or frequency. Taken together, these results suggest that CaMKIV signaling in vivo is not essential for homeostatic plasticity in pyramidal neurons in visual cortex, and argue against its role as a general regulator of firing rate set points since it does not regulate baseline mEPSC amplitude or intrinsic excitability. This lack of impact of CaMKIV knock-down on basal properties is consistent with a previous study showing no changes in the levels of pCREB, a downstream
CaMKIV effector, across five different brain regions in a constitutive CaMKIV knock-out mouse (Wei et al., 2002).

It is possible that the discrepancies between the effects of CaMKIV manipulation in vivo and in vitro are because of important experimental differences. Cultured rat cortical neurons were used between 7 and $14 \mathrm{~d}$ in vitro after being harvested on $\mathrm{P} 0$, while our ex vivo experiments were performed on mice ages P22 to P29 (Ibata et al., 2008; Joseph and Turrigiano, 2017), so it is possible that the pathways that regulate basal electrophysiological properties and homeostatic plasticity are strongly developmentally regulated, differentially expressed in mice and rats, or both. The duration of CaMKIV manipulation may be significant, as in vitro experiments have generally used 
either short pharmacological manipulations or brief genetic manipulations lasting only $24 \mathrm{~h}$ or so (Pratt et al., 2011; Joseph and Turrigiano, 2017) while our approach (conditional knock-down or viral expression in vivo) limited us to examining the impact of longer manipulations. It is possible that decreasing CaMKIV signaling for a week or more in vivo results in robust compensation. Like CaMKIV, CaMKI is activated by phosphorylation from CaMKK (Selbert et al., 1995) and can itself phosphorylate CREB at ser133 (Ginty et al., 1993), making it a particularly attractive candidate for a compensatory kinase; CaMKK has also been implicated in homeostatic plasticity (lbata et al., 2008; Goold and Nicoll, 2010; Pratt et al., 2011; Li et al., 2020), making it another attractive candidate. However, acute expression of dnCaMKIV in rodent hippocampal neurons in vivo also had no effect on synaptic properties (Marie et al., 2005), consistent with a lack of a role for in vivo CaMKIV signaling in regulating baseline excitability.

CaMKIV signaling has recently been implicated in the TTX-induced homeostatic broadening of action potentials caused by rebound following hyperpolarization in cultured hippocampal neurons (Li et al., 2020). In contrast, we found that $6 \mathrm{~d}$ of $\mathrm{MD}$, a protocol sufficient to trigger plasticity of intrinsic excitability, did not cause a broadening of action potentials triggered by direct depolarization. Similarly, CaMKIV knock-down did not affect action potential width in sham or monocularly deprived animals. This result suggests that CaMKIV signaling does not regulate action potential width ex vivo under physiological conditions, and that regulation of action potential width may not be a prominent feature of homeostatic plasticity of intrinsic excitability in cortical pyramidal neurons.

The function of CaMKIV signaling in cultured neurons may also be distinct from its function in vivo in L2/3 or L4. Firing rate set points are homeostatically maintained at the single neuron level in vivo (Hengen et al., 2016; Dhawale et al., 2017; Torrado Pacheco et al., 2021), but at the network level in cultured neurons (Slomowitz et al., 2015; Styr et al., 2019), which lack the stereotypical layered structure of intact cortex. This suggests that the specific connectivity of neurons may be critical for their expression of homeostatic plasticity. Since connectivity between neurons across and within layers in vivo is influenced by their developmental identity (Cadwell et al., $2019,2020)$, a loss of layer-specific identity in vitro may lead to aberrant relationships between CaMKIV expression (which is layer-specific in vivo), synaptic connectivity, and expression of homeostatic plasticity. It is possible that this disruption causes CaMKIV to assume a role in regulating neuronal excitability in vitro that it does not play in vivo.

The diverse effects of caCaMKIV expression on various intrinsic synaptic properties likely result from a complex redistribution of the expression of different ionic currents. In particular, the increase in action potential latency along with a decrease in spike frequency adaptation suggest an up-regulation of a transient $\mathrm{K}^{+}$current. The cumulative effects of these changes on the F-I curve of these neurons suggests that they may achieve a greater tendency toward an all or none response, where weak inputs are less likely to elicit a response while strong inputs elicit rapid firing.

However, care must be taken with the interpretation of these data from caCaMKIV, as expression of a constitutively active kinase could lead to off-target effects and overwhelm protein phosphatases. Indeed, it is possible that CaMKIV signaling plays no appreciable role in the maintenance of basal intrinsic excitability and synaptic input strength or homeostatic changes in these parameters in V1 pyramidal neurons in vivo, and that the effects of caCaMKIV expression are not physiologically relevant. This does not exclude a potential role for CaMKIV signaling in activity regulation in other excitatory or inhibitory neuron types, brain regions, or developmental stages; indeed, the effects of acute caCaMKIV expression in pyramidal neurons in the hippocampus in vivo differ from those seen here in visual cortex (Marie et al., 2005). Regardless of the explanation, our data are consistent with the relatively subtle phenotypes that have been reported in constitutive CaMKIV knock-out mice (Wei et al., 2002; Ko et al., 2006), and suggest that if CaMKIV signaling in the neocortex does modulate network stability in vivo, its loss can be robustly compensated.

\section{References}

Burrone J, O’Byrne M, Murthy VN (2002) Multiple forms of synaptic plasticity triggered by selective suppression of activity in individual neurons. Nature 420:414-418.

Cadwell CR, Bhaduri A, Mostajo-Radji MA, Keefe MG, Nowakowski TJ (2019) Development and arealization of the cerebral cortex. Neuron 103:980-1004.

Cadwell CR, Scala F, Fahey PG, Kobak D, Mulherkar S, Sinz FH, Papadopoulos S, Tan ZH, Johnsson P, Hartmanis L, Li S, Cotton RJ, Tolias KF, Sandberg R, Berens P, Jiang X, Tolias AS (2020) Cell type composition and circuit organization of clonally related excitatory neurons in the juvenile mouse neocortex. Elife 9: e52951.

Casanova E, Fehsenfeld S, Greiner E, Stewart AF, Schütz G (2002) Conditional mutagenesis of CamKIV. Genesis 32:161-164.

Chub N, O'Donovan MJ (1998) Blockade and recovery of spontaneous rhythmic activity after application of neurotransmitter antagonists to spinal networks of the chick embryo. J Neurosci 18:294306.

Cohen SM, Ma H, Kuchibhotla KV, Watson BO, Buzsáki G, Froemke RC, Tsien RW (2016) Excitation-transcription coupling in parvalbumin-positive interneurons employs a novel CaM kinase-dependent pathway distinct from excitatory neurons. Neuron 90:292-307.

Cruzalegui FH, Means AR (1993) Biochemical characterization of the multifunctional $\mathrm{Ca} 2+/$ calmodulin-dependent protein kinase type IV expressed in insect cells. J Biol Chem 268:26171- 26178.

Davis GW (2013) Homeostatic signaling and the stabilization of neural function. Neuron 80:718-728.

Desai NS, Rutherford LC, Turrigiano GG (1999) Plasticity in the intrinsic excitability of cortical pyramidal neurons. Nat Neurosci 2:515520.

Dhawale AK, Poddar R, Wolff SB, Normand VA, Kopelowitz E, Ölveczky BP (2017) Automated long-term recording and analysis of neural activity in behaving animals. Elife 6:e27702.

Ginty DD, Kornhauser JM, Thompson MA, Bading H, Mayo KE, Takahashi JS, Greenberg ME (1993) Regulation of CREB phosphorylation in the suprachiasmatic nucleus by light and a circadian clock. Science 260:238-241.

Goold CP, Nicoll RA (2010) Single-cell optogenetic excitation drives homeostatic synaptic depression. Neuron 68:512-528. 
Gorski JA, Talley T, Qiu M, Puelles L, Rubenstein JLR, Jones KR (2002) Cortical excitatory neurons and glia, but not GABAergic neurons, are produced in the Emx1-expressing lineage. J Neurosci 22:6309-6314.

Harley CM, Reilly MG, Stewart C, Schlegel C, Morley E, Puhl JG, Nagel C, Crisp KM, Mesce KA (2015) Compensatory plasticity restores locomotion after chronic removal of descending projections. J Neurophysiol 113:3610-3622.

Hengen KB, Torrado Pacheco A, McGregor JN, Van Hooser SD, Turrigiano GG (2016) Neuronal firing rate homeostasis is inhibited by sleep and promoted by wake. Cell 165:180-191.

Hrvatin S, Hochbaum DR, Nagy MA, Cicconet M, Robertson K, Cheadle L, Zilionis R, Ratner A, Borges-Monroy R, Klein AM, Sabatini BL, Greenberg ME (2018) Single-cell analysis of experience-dependent transcriptomic states in the mouse visual cortex. Nat Neurosci 21:120-129.

Ibata K, Sun Q, Turrigiano GG (2008) Rapid synaptic scaling induced by changes in postsynaptic firing. Neuron 57:819-826.

Joseph A, Turrigiano GG (2017) All for one but not one for all: excitatory synaptic scaling and intrinsic excitability are coregulated by CaMKIV, whereas inhibitory synaptic scaling is under independent control. J Neurosci 37:6778-6785.

Ko SW, Jia Y, Xu H, Yim SJ, Jang DH, Lee YS, Zhao MG, Toyoda H, Wu LJ, Chatila TA, Kaang BK, Zhuo M (2006) Evidence for a role of CaMKIV in the development of opioid analgesic tolerance. Eur $\mathrm{J}$ Neurosci 23:2158-2168.

Lambo ME, Turrigiano GG (2013) Synaptic and intrinsic homeostatic mechanisms cooperate to increase L2/3 pyramidal neuron excitability during a late phase of critical period plasticity. J Neurosci 33:8810-8819.

Li B, Suutari BS, Sun SD, Luo Z, Wei C, Chenouard N, Mandelberg NJ, Zhang G, Wamsley B, Tian G, Sanchez S, You S, Huang L, Neubert TA, Fishell G, Tsien RW (2020) Neuronal inactivity co-opts LTP machinery to drive potassium channel splicing and homeostatic spike widening. Cell 181:1547-1565.e15.

Lonze BE, Ginty DD (2002) Function and regulation of CREB family transcription factors in the nervous system. Neuron 35:605-623.

Ma H, Groth RD, Cohen SM, Emery JF, Li B, Hoedt E, Zhang G, Neubert TA, Tsien RW (2014) $\gamma$ CaMKII Shuttles $\mathrm{Ca}^{2+} / \mathrm{CaM}$ to the nucleus to trigger CREB phosphorylation and gene expression. Cell 159:281-294.

Marie H, Morishita W, Yu X, Calakos N, Malenka RC (2005) Generation of silent synapses by acute in vivo expression of CaMKIV and CREB. Neuron 45:741-752.

Moore AR, Richards SE, Kenny K, Royer L, Chan U, Flavahan K, Hooser SDV, Paradis S (2018) Rem2 stabilizes intrinsic excitability and spontaneous firing in visual circuits. Elife 7:e33092.

Prakash R, Yizhar O, Grewe BF, Ramakrishnan C, Wang N, Goshen I, Packer AM, Peterka DS, Yuste R, Schnitzer MJ, Deisseroth K (2012) Two-photon optogenetic toolbox for fast inhibition, excitation and bistable modulation. Nat Methods 9:1171-1179.
Pratt KG, Zimmerman EC, Cook DG, Sullivan JM (2011) Presenilin 1 regulates homeostatic synaptic scaling through Akt signaling. Nat Neurosci 14:1112.

Sakurai A, Katz PS (2009) Functional recovery after lesion of a central pattern generator. J Neurosci 29:13115-13125.

Schmitt JM, Wayman GA, Nozaki N, Soderling TR (2004) Calcium activation of ERK mediated by calmodulin kinase I. J Biol Chem 279:24064-24072.

Selbert MA, Anderson KA, Huang QH, Goldstein EG, Means AR, Edelman AM (1995) Phosphorylation and activation of Ca-calmodulin-dependent protein kinase IV by Ca-calmodulin-dependent protein kinase la kinase: phosphorylation of threonine 196 is essential for activation. J Biol Chem 270:17616- 17621.

Slomowitz E, Styr B, Vertkin I, Milshtein-Parush H, Nelken I, Slutsky M, Slutsky I (2015) Interplay between population firing stability and single neuron dynamics in hippocampal networks. Elife 4:e04378.

Styr B, Gonen N, Zarhin D, Ruggiero A, Atsmon R, Gazit N, Braun G, Frere S, Vertkin I, Shapira I, Harel M, Heim LR, Katsenelson M, Rechnitz O, Fadila S, Derdikman D, Rubinstein M, Geiger T, Ruppin E, Slutsky I (2019) Mitochondrial regulation of the hippocampal firing rate set point and seizure susceptibility. Neuron 102:1009-1024.e8.

Thoby-Brisson M, Simmers J (1998) Neuromodulatory inputs maintain expression of a lobster motor pattern-generating network in a modulation-dependent state: evidence from long-term decentralization in vitro. J Neurosci 18:2212-2225.

Ting JT, Daigle TL, Chen Q, Feng G (2014) Acute brain slice methods for adult and aging animals: application of targeted patch clamp analysis and optogenetics. Methods Mol Biol 1183:221-242.

Torrado Pacheco A, Bottorff J, Gao Y, Turrigiano GG (2021) Sleep promotes downward firing rate homeostasis. Neuron 109:530544.e6.

Turrigiano G (2012) Homeostatic synaptic plasticity: local and global mechanisms for stabilizing neuronal function. Cold Spring Harb Perspect Biol 4:a005736.

Turrigiano GG, Nelson SB (2004) Homeostatic plasticity in the developing nervous system. Nat Rev Neurosci 5:97-107.

Turrigiano GG, Leslie KR, Desai NS, Rutherford LC, Nelson SB (1998) Activity-dependent scaling of quantal amplitude in neocortical neurons. Nature 391:892-896.

Wayman GA, Kaech S, Grant WF, Davare MA, Impey S, Tokumitsu H, Nozaki N, Banker G, Soderling TR (2004) Regulation of axonal extension and growth cone motility by calmodulin-dependent protein kinase I. J Neurosci 24:3786-3794.

Wayman GA, Tokumitsu H, Davare MA, Soderling TR (2011) Analysis of CaM-kinase signaling in cells. Cell Calcium 50:1-8.

Wei F, Qiu C-S, Liauw J, Robinson DA, Ho N, Chatila T, Zhuo M (2002) Calcium-calmodulin-dependent protein kinase IV is required for fear memory. Nat Neurosci 5:573.

Zhang W, Linden DJ (2003) The other side of the engram: experience-driven changes in neuronal intrinsic excitability. Nat Rev Neurosci 4:885-900. 